\title{
Social polymorphism is favoured by the co-evolution of dispersal with social behaviour
}

\author{
Charles Mullon ${ }^{\star}{ }^{\star}$, Laurent Keller` and Laurent Lehmann
}

\begin{abstract}
Dispersal determines gene flow among groups in a population and so plays a major role in many ecological and evolutionary processes. As gene flow shapes kin structure, dispersal is important to the evolution of social behaviours that influence reproduction within groups. Conversely, dispersal depends on kin structure and social behaviour. Dispersal and social behaviour therefore co-evolve, but the nature and consequences of this interplay are not well understood. Here, we show that it readily leads to the emergence of two social morphs: a sessile, benevolent morph expressed by individuals who tend to increase the reproduction of others within their group relative to their own; and a dispersive, self-serving morph expressed by individuals who tend to increase their own reproduction. This social polymorphism arises due to a positive linkage between the loci responsible for dispersal and social behaviour, leading to benevolent individuals preferentially interacting with relatives and selfserving individuals with non-relatives. We find that this linkage is favoured under a large spectrum of conditions, suggesting that associations between dispersal and other social traits should be common in nature. In line with this prediction, dispersers across a wide range of organisms have been reported to differ in their social tendencies from non-dispersers.
\end{abstract}

D ispersal, the movement away from natal habitat to reproduce, is an important step in the life history of most organisms ${ }^{1,2}$. At the population level, dispersal patterns shape kin structure, which determines whether individuals interact and compete with relatives. This, in turn, influences the evolution of social behaviour such as helping or aggression ${ }^{3,4}$. At the same time, dispersal decisions are often influenced by kin and social interactions ${ }^{1,5-7}$, resulting in the co-evolution among dispersal between groups and social behaviours within groups ${ }^{8-16}$. However, the consequences of this coevolution for within-species behavioural diversity (see, for example, ref. ${ }^{17}$ ) remain elusive. Here, we model the co-evolution between unconditional dispersal and social behaviours, and show that it readily leads to a stable genetic and social polymorphism, whereby individuals who disperse behave differently from non-dispersers.

To model social interactions within groups and dispersal between groups, we assume that the population is structured according to the infinite-island model ${ }^{18,19}$, in which individuals belong to local groups and interact socially only with other locals. As a baseline, we assume that groups are of fixed size $N$, and that individuals reproduce asexually and then die so that generations do not overlap. An offspring either remains in its natal group (with probability $1-d$ ), or disperses to another randomly chosen one (with probability $d$ ) and survives dispersal with probability $1-c_{\mathrm{d}}$. Social interactions are modelled with a classical matrix game ${ }^{20}$ : individuals randomly pair up within their group and each independently chooses between two actions denoted B (with probability $z$ ) and M (with probability $1-z)$. Depending on the action of each player, each reaps a material payoff that in turn linearly increases its fecundity. Without loss of generality, we assume that when both play $M$, they obtain no payoff (see Methods for more details on the game). If one plays B and the other plays $\mathrm{M}$, the $\mathrm{B}$ player gets the (direct) benefit $B_{\mathrm{D}}$ and the $\mathrm{M}$ player gets the (indirect) benefit $B_{\mathrm{I}}$. We assume that $B_{\mathrm{I}}-B_{\mathrm{D}}>0$, which means that an individual who plays $B$ more often that its partner increases its partner's fecundity relative to its own, and conversely, an individual who plays $M$ more often decreases its partner's fecundity. We therefore refer to action $B$ as benevolent and $M$ as self-serving. Finally, if they both play $\mathrm{B}$, they each get $B_{\mathrm{D}}+B_{\mathrm{I}}-S$, where $S>0$ is the antagonistic synergy of benevolence; that is, $S$ captures the degree with which returns diminish with the number of individuals adopting the benevolent action $\mathrm{B}$ in a pair.

\section{Results}

Co-evolutionary dynamics of dispersal and social behaviour. First, we study mathematically the co-evolution of the probability $d$ of dispersing with the probability $z$ of adopting the benevolent action $\mathrm{B}$ when they are encoded by two linked loci that experience rare mutations with small quantitative effects ${ }^{21}$ (see Methods). In agreement with previous results, the population first evolves gradually to converge towards an equilibrium for both traits: dispersal converges to an equilibrium $0<d^{*} \leq 1$ that depends on the cost $c_{\mathrm{d}}$ of dispersal and group size ${ }^{5,22}$ (Fig. 1), while the probability $z$ of adopting the benevolent action $\mathrm{B}$ converges to $0 \leq z^{*}=B_{\mathrm{D}} / S \leq 1$ (provided $0 \leq B_{\mathrm{D}} \leq S$, Supplementary Note 1.1 for details $)^{23}$. Once the population has converged to the equilibrium $\left(d^{*}, z^{*}\right)$ for both dispersal and benevolence, the population either is maintained at this equilibrium by stabilizing selection (that is, the population is uninvadable by any alternative strategy) and remains monomorphic, or undergoes disruptive selection and becomes polymorphic.

Mathematical analysis reveals that disruptive selection occurs under a wide range of model parameters (Fig. 2), and that it leads to the emergence of two morphs: a more benevolent, sessile morph; and a more self-serving, dispersive morph (Supplementary Notes 1.2 and 1.3). To understand why selection favours these two morphs, consider an individual that expresses the benevolent, sessile morph. Such an individual tends to preferentially interact with related individuals of the same morph, and so its benevolence is preferentially directed towards relatives. Conversely, an individual from the dispersive morph preferentially interacts with less related individuals, and thus benefits from being self-serving. Polymorphism therefore arises due to the combined effects of dispersal on kin interaction and social behaviour on neighbours' fitness. In line with this, when only one trait (dispersal or benevolence) evolves and the other is fixed, the population remains monomorphic for all model parameters (Supplementary Note 1.2). 


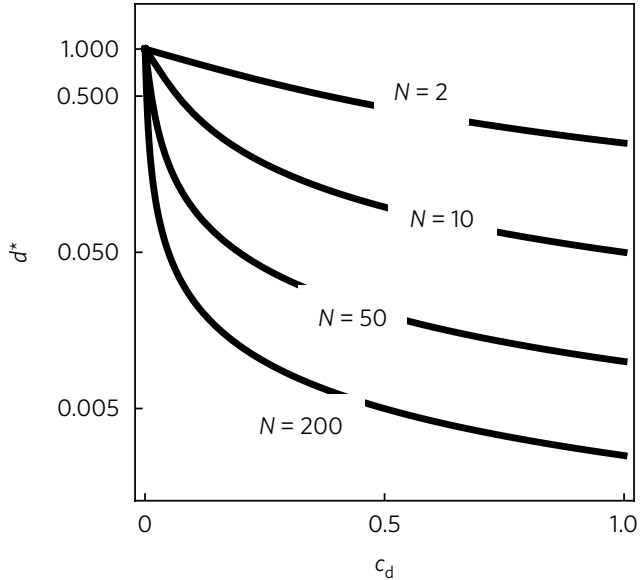

Fig. 1 | Equilibrium dispersal. Dispersal equilibrium $d^{\star}$ in terms of the cost of dispersal, $c_{d}$, for different group sizes $N$ (see Supplementary equation (3) in Supplementary Note 1.1 for the mathematical expression).

To check our mathematical analyses and investigate the long-term effects of disruptive selection, we ran individual-based simulations under conditions that should lead to polymorphism. As predicted, the population first converges to the interior equilibrium for dispersal $d^{*}$ and the probability $z^{*}$ to adopt benevolent action B, and splits into two morphs, a more benevolent, sessile morph, and a more self-serving, dispersive morph (Fig. 3a). Competition among the two morphs then creates a positive feedback that favours more extreme variants. The population eventually stabilizes for two highly differentiated genetic morphs, resulting in a strong association between dispersal and social behaviour (Fig. 3b).

To test whether an association between dispersal and social behaviour also emerges when selection is stabilizing, we ran simulations under such conditions. As predicted by our analysis, the phenotypic distribution in the population remains centred around the uninvadable equilibrium (Fig. 3c). A negative association among dispersal and benevolent behaviour also emerges, but it is weaker than when selection is disruptive (compare Fig. $3 b$ with $3 c$ ).

Relaxing baseline model assumptions. Three important assumptions made in the baseline model are that generations do not overlap, that group size is fixed and that the population is structured according to the standard island model. We relaxed the first assumption by performing a mathematical analysis of dispersal and social behaviour co-evolution when a single individual is replaced at each generation in each group. This analysis reveals that polymorphism is also often favoured in this scenario. In fact, compared with our baseline model, polymorphism is favoured for an even greater diversity of

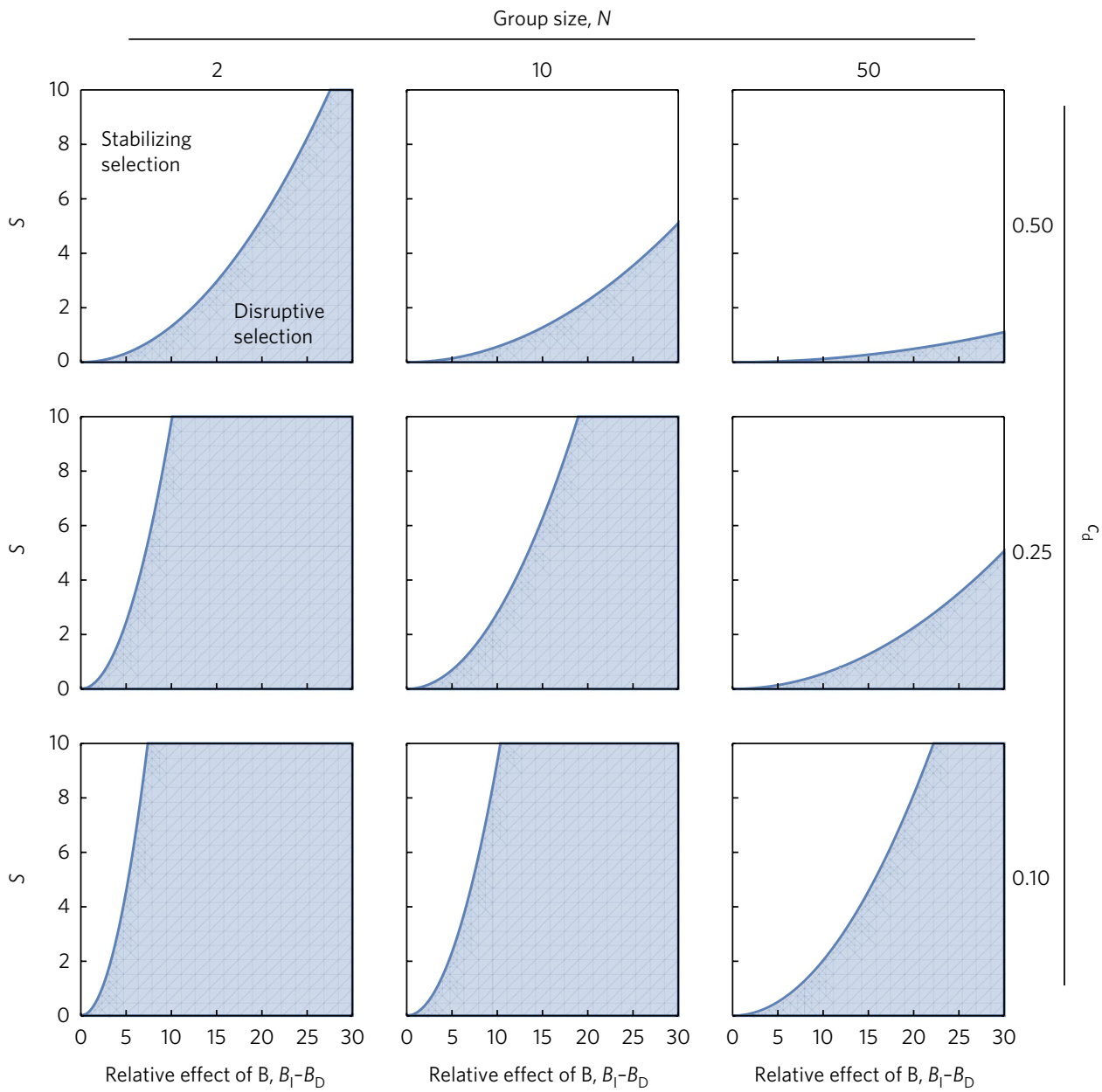

Fig. 2 | Disruptive selection when dispersal and social behaviour co-evolve. The parameter region under which disruptive selection leads to polymorphism at the equilibrium $\left(d^{\star}, z^{\star}\right)$ (shaded region, computed from Supplementary equation (17) in Supplementary Note 1.3, here shown with individual fecundity at the equilibrium set to 1 ). Disruptive selection is therefore favoured when groups are small; dispersal cost $c_{\mathrm{d}}$ is low; antagonistic synergy $S$ is weak; and benevolence has large relative effects (that is, $B_{1}-B_{D}$ is large). 
a

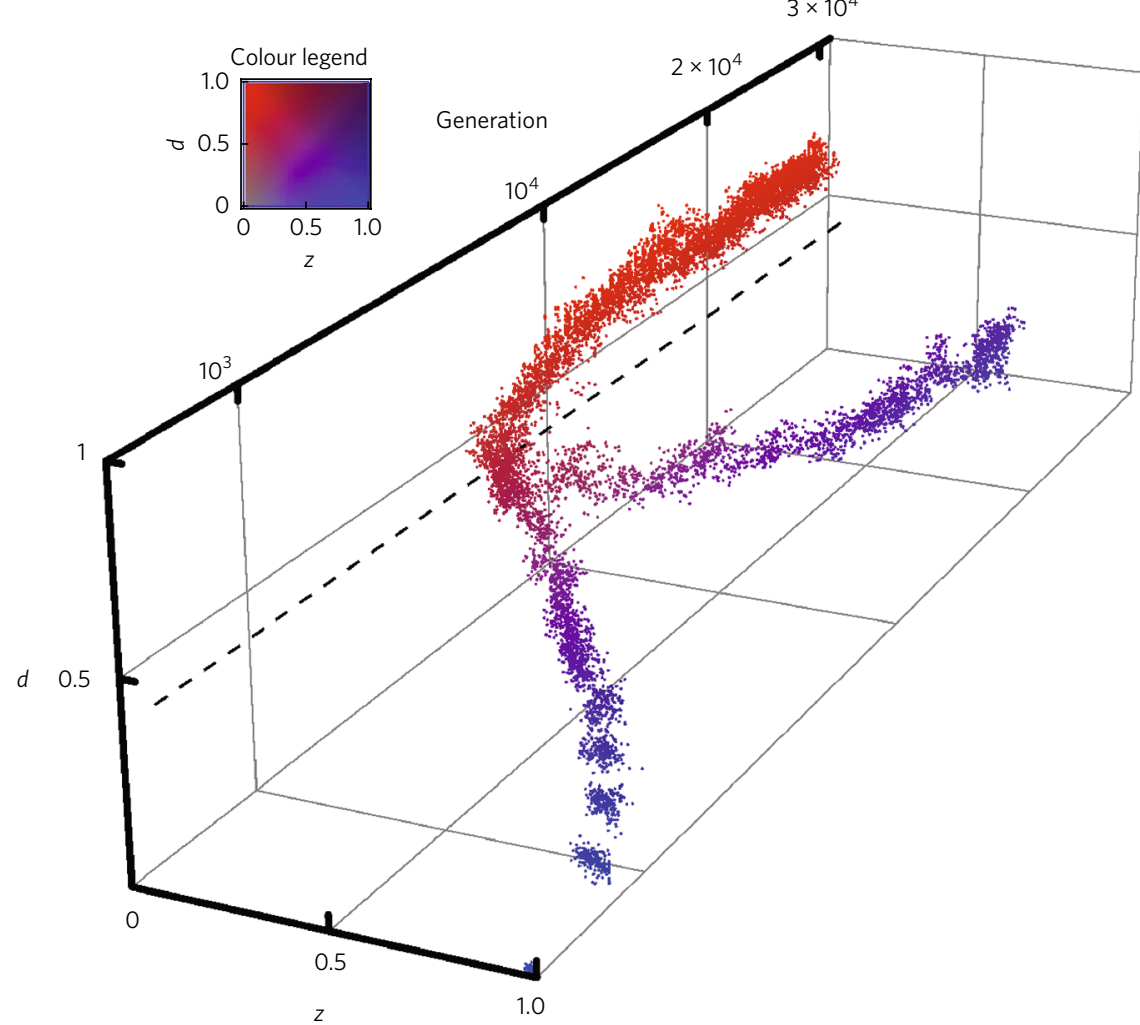

b

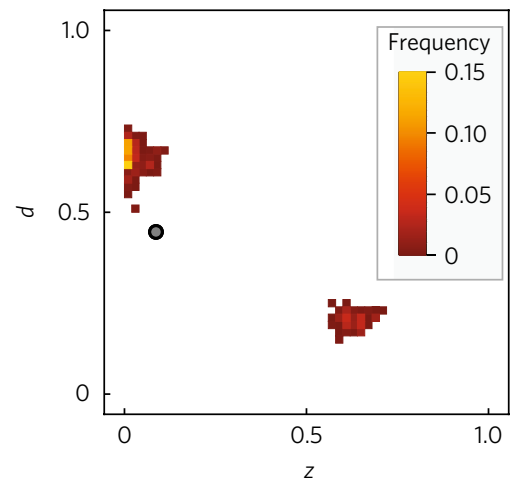

C

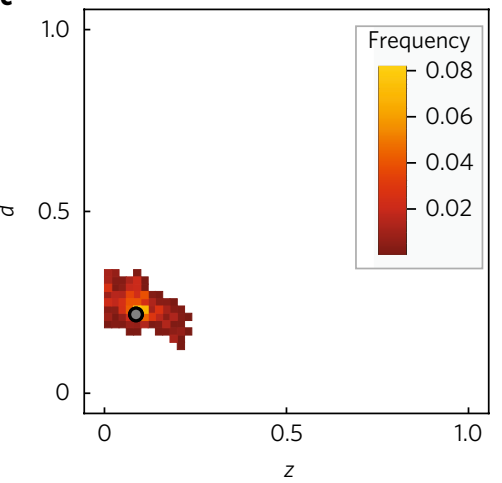

Fig. 3 | The emergence and maintenance of social morphs when dispersal and social behaviour co-evolve. a, Dispersal $d$ and benevolence $z$ in a simulated population of 10,000 individuals (trait values of 300 individuals randomly sampled every 500 generations; see the colour legend for the colouring scheme, and Supplementary Note 3.1 for details on the simulations) and predicted interior equilibrium (dashed black line, with $B_{D}=0.05, B_{1}=1.95, S=0.55, N=8$, $c_{d}=0.1$ and baseline fecundity set to 1 ). $\mathbf{b}$, Phenotypic distribution of the whole population at generation $3 \times 10^{4}$ (the same parameters as in $\mathbf{a}$; the predicted interior equilibrium is shown by the grey disc). c, Phenotypic distribution of the whole population at generation $3 \times 10^{4}$ under balancing selection (with $c_{d}=0.25$; the other parameters are the same as in $\mathbf{a}$; the predicted interior equilibrium is shown by the grey disc).

payoff variables, which means that a greater diversity of social behaviours may become associated with dispersal when generations overlap (Supplementary Note 2). We next relaxed the second and third assumptions by letting group size fluctuate (with regulation through local competition), and by isolating groups by distance (instead of an island model). For both conditions, we compared the outcomes of three simulation experiments: we fixed dispersal and let only social behaviour evolve; we fixed social behaviour and let only dispersal evolve; and we allowed both traits to co-evolve. These experiments show that distinct social morphs emerge as a result of disruptive selection, but as under the baseline scenario, this is true only when both traits co-evolve (Supplementary Figs. 1 and 2).

We also tested the importance of genetic linkage for the emergence of highly differentiated social morphs by studying the effects of various levels of recombination between the loci that control dispersal and social behaviour (Supplementary Note 3.2). This revealed that the emergence of distinct morphs depends on the level of recombination. As recombination increases, maladapted morphs that are benevolent and dispersive or self-serving and sessile increase in frequency (Fig. 4a). Beyond a threshold, recombination prevents polymorphism altogether and the population remains monomorphic (Fig. 4a). This is because strong recombination breaks the positive genetic linkage among the dispersal and social behaviour loci that is necessary for benevolent individuals to preferentially direct their benevolence towards relatives, and self-serving individuals to compete with non-relatives.

The evolution and signature of a supergene for dispersal and social behaviour. Since disruptive selection promotes an association among dispersal and social behaviour, it should also promote a genetic architecture that makes this association heritable ${ }^{24}$. We tested this by adding a third locus that controls recombination and let it evolve by introducing two alleles that mutate from one another, one recessive wild type that codes for a recombination probability of $1 / 2$ and one dominant mutant that stops recombination (Supplementary Note 3.3). Starting with a wild-type population at the predicted equilibrium $\left(d^{\star}, z^{\star}\right)$ for dispersal and social behaviour, the mutant allele at the recombination modifier locus eventually invades so that recombination is shut down, which then permits the emergence of distinct morphs (Fig. 4b). Disruptive selection therefore leads to the genetic integration of dispersal and social behaviours to form a 'supergene ${ }^{25}$, which allows benevolent individuals to preferentially interact with relatives, and self-serving individuals with non-relatives. This type of kin association through genetic and spatial assortment may constitute a first step towards conditional dispersal ${ }^{10}$ or conditional social behaviours ${ }^{8,11,26}$, which allow individuals to fine-tune their behaviours towards relatives.

Finally, we studied the population genetic signatures associated with the emergence of this social polymorphism. We first calculated the degree of genetic differentiation $\left(F_{\mathrm{ST}}\right)$ among morphs at the locus responsible for social behaviour (the 'selected locus') and at a neutral, unlinked locus. Genetic differentiation among morphs at the selected locus is much greater than at a neutral locus (Fig. 5), which is unsurprising since social behaviour is genetically determined. Second, we looked at the degree of genetic differentiation among groups within each social morph, and found that differentiation among groups is greater within the benevolent 
a

Recombination probability
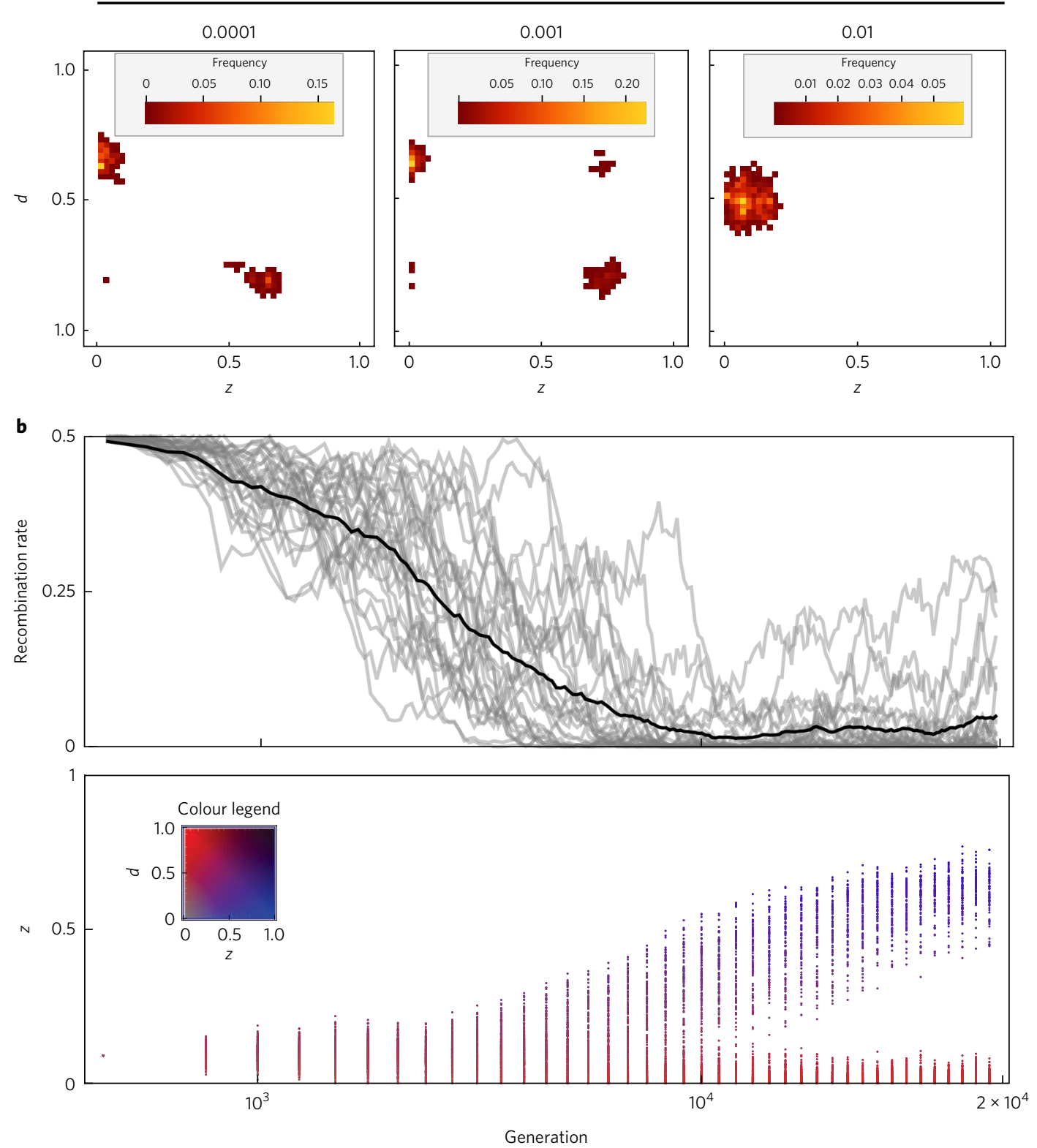

Fig. 4 | The effect and evolution of genetic linkage between dispersal and social behaviour loci. a, Phenotypic distribution of the whole population at generation $2 \times 10^{4}$ when the recombination probability is fixed (the recombination value is shown above the graphs, with $B_{\mathrm{D}}=0.05, B_{1}=1.95, S=0.55$, $N=8, c_{d}=0.1$ and baseline fecundity set to 1 ; see Supplementary Note 3.2 for details). $\mathbf{b}$, Upper panel: effective recombination rate in the population (number of recombination events/population size) every 100 generations when recombination evolves in a population of 10,000 individuals (for 30 replicates; each replicate is shown by a grey line; the average recombination rate over replicates is shown in black). Lower panel: benevolence $z$ of 300 individuals randomly sampled every 500 generations across all 30 replicates (see the colour legend for the colouring scheme). Polymorphism arose in all 30 replicates.

morph than the self-serving one at the selected locus (Fig. 5). This pattern arises because the benevolent morph has lower dispersal tendencies than the self-serving one. Interestingly, differentiation among groups is also greater within the benevolent morph at the neutral locus (Fig. 5). The effect of dispersal differences between the social morphs therefore extends beyond the selected locus and creates detectable patterns of genetic differentiation at neutral unlinked loci.

\section{Discussion}

These analyses reveal that the co-evolution of dispersal and social behaviour favours the emergence of a social polymorphism and dispersal syndrome. Clear predictions can be extrapolated from our results. The first is that phenotypic associations between dispersal and social behaviour should be common. This prediction supported by findings in multiple organisms ranging from protozoa to primates where an intraspecific association between dispersal and social behaviour has been found (Table 1 , see also refs ${ }^{10,27-29}$ for reviews). For example, in the ciliate Tetrahymena thermophila, laboratory studies have shown that strains that are more cooperative disperse at a lower rate than less cooperative strains at intermediate population densities ${ }^{30}$. Similarly, in wild populations of prairie voles Microtus pennsylvanicus, individuals that disperse tend to be more aggressive than those who do not ${ }^{31}$. 


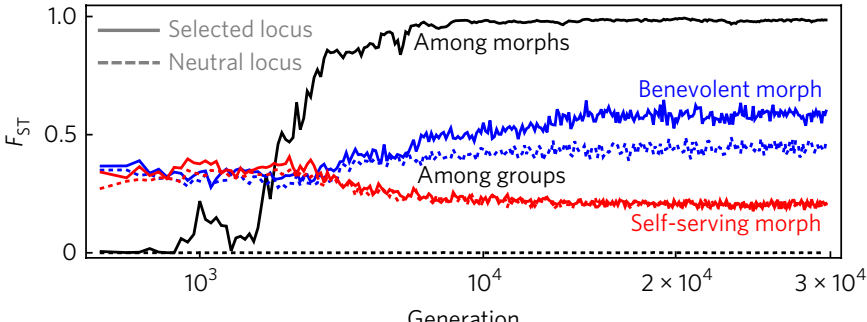

Generation

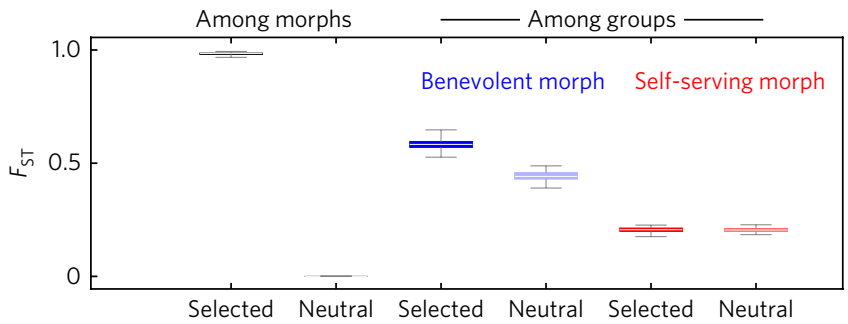

Fig. 5 | Patterns of genetic differentiation. Upper panel: $F_{\mathrm{ST}}$ values calculated every 100 generations for a population initially monomorphic for its predicted equilibrium that becomes polymorphic when the loci for dispersal and social behaviours are genetically linked (see Supplementary Note 3.4 for information on the $F_{\mathrm{ST}}$ calculations; simulation parameters: $B_{\mathrm{D}}=0.05, B_{1}=1.95, S=0.55, N=8, C_{d}=0.1$ and baseline fecundity set to 1 ). Black: genetic differentiation among morphs at the selected locus (full line) and at an unlinked neutral locus (dashed line). Blue: genetic differentiation among groups within the benevolent morph at the selected locus (full line) and at an unlinked neutral locus (dashed line). Red: genetic differentiation among groups within the self-serving morph at the selected locus (full line) and at an unlinked neutral locus (dashed line). Lower panel: $F_{\mathrm{ST}}$ values between generations $1.5 \times 10^{4}$ and $3 \times 10^{4}$ among morphs (black) and among groups within benevolent (blue) and self-serving (red) individuals at the selected (darker shade) and an unlinked neutral (lighter shade) locus (the coloured error bars show the 50\% confidence interval, and the black error bars show the distribution range).

Our prediction that benevolence and dispersal propensity should be associated at the phenotypic level aligns with the predictions stemming from models of conditional behaviours ${ }^{8,10,11}$. When individuals can condition their social behaviour on whether they have dispersed or not, selection favours increased self-servingness in dispersers and benevolence in non-dispersers ${ }^{11}$, also creating a negative phenotypic association between benevolence and dispersal behaviours. Although studies on conditional behaviours ${ }^{8,10,11}$ have not tested whether genetic polymorphism would also emerge due to disruptive selection, it is unlikely because conditional behaviour already allows one to behave more benevolently towards relatives, and conditional behaviours typically reduce disruptive selection $^{32}$. The evolution of conditional behaviour thus results in a phenotypic but not a genetic association among dispersal and social behaviours.

Here, the social polymorphism we report is underlain by a genetic association among dispersal and social behaviours. Previous models of the co-evolution of unconditional dispersal and cooperation have also found that genetic polymorphism can emerge in asexual haploids ${ }^{14-16}$. However, these models assume that cooperation has nonlinear fitness effects such that polymorphism can arise when cooperation is evolving but the rate of dispersal is fixed. The importance of the co-evolution of dispersal and social behaviours for polymorphism is therefore unclear from these models. By contrast, social polymorphism in our model emerges only when dispersal and social behaviours co-evolve.

Importantly, our analyses suggest that selection also leads to the genetic integration of dispersal and social behaviours into a
Table 1 | Species in which a phenotypic association between dispersal and social behaviour has been described

\begin{tabular}{lll} 
Social behaviour & $\begin{array}{l}\text { Association } \\
\text { with dispersal }\end{array}$ & Species \\
\hline $\begin{array}{l}\text { Aggressiveness, } \\
\text { 'self-servingness' }\end{array}$ & Positive & $\begin{array}{l}\text { Sialia mexicana, Clethrionomys } \\
\text { rufocanus, Microtus pennsylvanicus, } \\
\text { Myodes glareolus, Rhabdomys } \\
\text { pumilio, Neolamprologus pulcher, } \\
\text { Mus musculus domesticus, Macaca } \\
\text { mulatta, Drosophila melanogaster }\end{array}$ \\
& & $\begin{array}{l}\text { Picoides borealis, Lacerta vivipara, } \\
\text { Pro-social, helping, Negative } \\
\text { cooperation, } \\
\text { 'Benevolence' }\end{array}$ \\
& & $\begin{array}{l}\text { Tetrahymena thermophila, Uta } \\
\text { stansburiana, Heterocephalm glaber, } \\
\text { Apus melba }\end{array}$ \\
\hline
\end{tabular}

See Supplementary Table 1 for references to the literature.

single Mendelian genetic element. This brings us to our second prediction: dispersal and social behaviour should be genetically associated when polymorphism is present. Data to test this prediction are scarce because few studies combine dispersal, behavioural and heritability assays. One notable exception is found in western bluebirds Sialia mexicana, for which a multi-generational pedigree analysis revealed that dispersal and social behaviour are genetically associated, such that dispersive males are more likely to produce aggressive offspring and non-dispersive males are more likely to produce non-aggressive offspring ${ }^{33}$. A similar pattern of positive genetic association among dispersal and aggressiveness has been observed in two species of wild house mice Mus musculus musculus ${ }^{34}$ and Mus domesticus ${ }^{35}$. Lines of Drosophila melanogaster that have been selected for greater dispersal propensity also exhibit elevated aggressiveness ${ }^{36}$, which shows that genetic correlations among dispersal and aggressiveness are also present in this species. Conversely, in the colony-breeding Alpine swift Apus melba, dispersal and cooperative defence against human intrusion are negatively associated both at the phenotypic and genetic level ${ }^{37}$.

Our prediction of a genetic association among dispersal and social behaviour could be tested further in socially polymorphic species, such as social spiders or halictine bees. Between Anelosimus spider species, natal dispersal tends to be negatively associated with social living and behaviour ${ }^{38}$. It would be interesting to test whether this association also occurs within species. In particular, in the social spider Anelosimus studiosus, an aggressive morph co-exists with a docile one ${ }^{39}$, and evidence already suggests that expressing the aggressive morph is heritable ${ }^{40}$ and associated with other social behaviours ${ }^{41}$, but an association between dispersal and aggressiveness has not yet been studied. Direct evidence for such an association would require dispersal and heritability assays that can be challenging. Alternatively, indirect evidence could be provided by patterns of genetic differentiation $\left(F_{\mathrm{ST}}\right)$ among groups. In particular, our model suggests that a genetic association between dispersal and aggressiveness should lead to greater between-group genetic differentiation (at both selected and neutral loci) within the docile morph than within the aggressive morph (Fig. 5).

Eusocial species, which typically exhibit rich and variable patterns of dispersal and social behaviours, also provide a good model to test our prediction that dispersal and social behaviour should be genetically associated. Many ant species show a dispersal syndrome that associates dispersal with social organization. Queens either disperse far away from their natal nest and form singlequeen (monogyne) colonies, or disperse short distances and form multiple-queen (polygyne) colonies ${ }^{42}$. These two morphs are 
frequently found in the same population and show little genetic differentiation, suggesting extensive gene flow among them ${ }^{42-45}$. In line with the predictions of our model, individuals from monogyne colonies exhibit high intra-specific aggression towards nonnestmates while individuals from polygyne colonies are much less aggressive $e^{42}$. The genetic underpinning of social organization has been uncovered in two ant species, Solenopsis invicta ${ }^{44}$ and Formica selysi ${ }^{45}$. Remarkably, in both cases, the social polymorphism and dispersal syndrome is controlled by a large nonrecombining region that has independently arisen in each species, which suggests that integration of dispersal and social behaviour into a supergene can readily occur in nature.

Our simple model, of course, cannot explain all associations among dispersal and social behaviour, which can be influenced by many other factors (for example, in species with a social hierarchy, such as meerkats ${ }^{46}$, it may be beneficial to be more submissive when dispersing into a foreign group, so that we may expect benevolent behaviours to be positively associated with dispersal; see also refs ${ }^{27,29}$ ). Yet, the selection that associates dispersal and social behaviour in our model will influence evolution under most ecological settings because it depends only on kin structure, which, due to limited dispersal and the spatial scale of social interactions, is ubiquitous in nature ${ }^{47}$. While current data support the notion that individuals who disperse behave towards conspecifics in a way that is different from non-dispersers, further pedigree and genomic analyses will provide a better picture of how associations among dispersal and social behaviour are genetically constructed.

\section{Methods}

Matrix game. We use a pairwise symmetric matrix game ${ }^{20}$ to model social interactions within groups. Without loss of generality, we assume that the game is described by the following payoff matrix

$$
\begin{array}{ccc} 
& \mathrm{B} & \mathrm{M} \\
\mathrm{B} & B_{\mathrm{I}}+B_{\mathrm{D}}-S & B_{\mathrm{D}} \\
\mathrm{M} & B_{\mathrm{I}} & 0
\end{array}
$$

whose entries give the payoff to a focal row player (that is, when both play $\mathrm{B}$, the focal obtains $B_{\mathrm{I}}+B_{\mathrm{D}}-S$; when the focal plays $\mathrm{B}$ and the partner plays $\mathrm{M}$ it obtains $B_{\mathrm{D}}$; in the reverse situation, the focal obtains $B_{\mathrm{I}}$; and when both partners play $\mathrm{M}$, the focal obtains 0 ).

The payoff matrix (equation (1)) entails that the average payoff to a focal player who adopts action $\mathrm{B}$ with probability $z_{1}$ against a partner who adopts this action with probability $z_{2}$ is

$$
\pi\left(z_{1}, z_{2}\right)=\left(B_{\mathrm{I}}+B_{\mathrm{D}}-S\right) z_{1} z_{2}+B_{\mathrm{D}} z_{1}\left(1-z_{2}\right)+B_{\mathrm{I}}\left(1-z_{1}\right) z_{2}
$$

We assume that the payoff that an individual receives increases its fecundity linearly, in which case the fecundity of the partner, relative to the fecundity of the focal player, can be written as

$$
\frac{f_{0}+\pi\left(z_{2}, z_{1}\right)}{f_{0}+\pi\left(z_{1}, z_{2}\right)}=1+\frac{\left(B_{\mathrm{I}}-B_{\mathrm{D}}\right)\left(z_{1}-z_{2}\right)}{f_{0}+\pi\left(z_{1}, z_{2}\right)}
$$

where $f_{0}$ is a baseline fecundity that ensures that fecundity is positive. Equation (3) shows that when $B_{\mathrm{I}}-B_{\mathrm{D}}>0$ and the focal is more likely to express $\mathrm{B}$ than its partner $\left(z_{1}-z_{2}>0\right)$, this results in an increase of the partner's fecundity relative to that of the focal. Conversely, when the focal is less likely to express B than its partner $\left(z_{1}-z_{2}<0\right)$, this results in a decrease of its partner's fecundity relative to its own. The quantity $B_{\mathrm{I}}-B_{\mathrm{D}}$ can therefore be thought of as the relative fecundity effect of action $\mathrm{B}$.

We assume throughout that $B_{\mathrm{I}}-B_{\mathrm{D}}>0$, so that expressing action $\mathrm{B}$ increases the fecundity of its recipients relative to its actor, and we therefore call behaviour $\mathrm{B}$ 'benevolent'. Conversely, expressing $\mathrm{M}$ increases the fecundity of its actor relative to its recipient, and we call behaviour $M$ 'self-serving. The condition that $B_{\mathrm{I}}-B_{\mathrm{D}}>0$ includes well-known social dilemma games. For instance, when $B_{\mathrm{I}}>B_{\mathrm{I}}+B_{\mathrm{D}}-S>B_{\mathrm{D}}>0$, B behaviour is 'Dove' in the Hawk-Dove game, or 'Cooperate' in the Snow-Drift (or Volunteers' dilemma) game. When $B_{\mathrm{I}}>B_{\mathrm{I}}+B_{\mathrm{D}}-S>0>B_{\mathrm{D}}$, $\mathrm{B}$ is 'Cooperate' in the Prisoner's dilemma game. Behaviour $\mathrm{B}$ therefore generally encompasses cooperative and altruistic behaviours but not necessarily. Whether behaviour B is cooperative or altruistic sensu evolutionary biology depends on its fitness effects ${ }^{19,48}$, which themselves depend on population structure and life cycle.
Evolutionary invasion analysis in the island model. Invasion and the average mutant growth rate. In the infinite-island model, the fate of a mutation that codes for a rare mutant phenotype $\mathbf{x}_{\mathrm{m}}=\left(z_{\mathrm{m}}, d_{\mathrm{m}}\right)$ when the resident population has phenotype $\mathbf{x}=(z, d)$ can be deduced from the geometric growth rate $W\left(\mathbf{x}_{\mathrm{m}}, \mathbf{x}\right)$ of that mutation ${ }^{49,50}$, which is the time-averaged mean cumulative growth over different replicates or sample paths of the invasion dynamics. If the geometric growth rate is less than or equal to $1\left(W\left(\mathbf{x}_{\mathrm{m}}, \mathbf{x}\right) \leq 1\right)$, then the mutation will eventually go extinct in the population; otherwise it may persist indefinitely ${ }^{16}$. When the mutant and residents differ by only a small amount (that is, the Euclidean distance between $\mathbf{x}_{\mathrm{m}}$ and $\mathbf{x}$ is small, $\left\|\mathbf{x}_{\mathrm{m}}-\mathbf{x}\right\| \ll 1$ ), the growth rate can be approximated by Taylor expanding $W\left(\mathbf{x}_{\mathrm{m}}, \mathbf{x}\right)$ close to resident phenotype $\mathbf{x}$,

$$
W\left(\mathbf{x}_{\mathrm{m}}, \mathbf{x}\right) \approx 1+\left(\mathbf{x}_{\mathrm{m}}-\mathbf{x}\right)^{\mathrm{T}} \mathbf{s}(\mathbf{x})+\left(\mathbf{x}_{\mathrm{m}}-\mathbf{x}\right)^{\mathrm{T}} \mathbf{H}(\mathbf{x})\left(\mathbf{x}_{\mathrm{m}}-\mathbf{x}\right)
$$

where $\mathbf{s}(\mathbf{x})$ is a $2 \times 1$ vector and $\mathbf{H}(\mathbf{x})$ is a $2 \times 2$ matrix that respectively give the firstand second-order effects of selection ${ }^{16,50}$, which can be used to infer the adaptive dynamics of both traits. We detail $\mathbf{s}(\mathbf{x})$ and $\mathbf{H}(\mathbf{x})$ below.

Directional selection in the infinite-island model. When mutations are rare with weak phenotypic effects, the population first evolves under directional selection whereby selected new mutations rapidly sweep the population before a new mutation arises, so that the population 'jumps' from one monomorphic state to another ${ }^{21}$. The direction of evolution under directional selection is indicated by the selection gradient vector

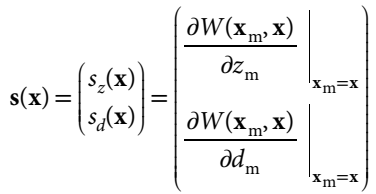

In the infinite-island model, the selection gradient on trait $u \in\{z, d\}$, which captures the directional coefficient of selection on trait $u$, has been shown (for example, equation (12) of ref. ${ }^{16}$ ) to be equal to

$$
s_{u}(\mathbf{x})=\left.\frac{\partial w\left(\mathbf{x}_{1}, \mathbf{x}_{-1}, \mathbf{x}\right)}{\partial u_{1}}\right|_{\substack{\mathbf{x}_{1}=\mathbf{x} \\ \mathbf{x}_{-1}=\mathbf{x}}}+\left.(N-1) r_{2}(\mathbf{x}, \mathbf{x}) \frac{\partial w\left(\mathbf{x}_{1}, \mathbf{x}_{-1}, \mathbf{x}\right)}{\partial u_{2}}\right|_{\substack{\mathbf{x}_{1}=\mathbf{x} \\ \mathbf{x}_{-1}=\mathbf{x}}}
$$

where $w\left(\mathbf{x}_{1}, \mathbf{x}_{-1}, \mathbf{x}\right)$ is the individual fitness of a focal individual that we arbitrarily label as individual 1 (that is, the expected number of adult offspring produced by individual 1 ), when it has phenotype $\mathbf{x}_{1}=\left(z_{1}, d_{1}\right)$, his $N-1$ neighbours have phenotypes $\mathbf{x}_{-1}=\left(\mathbf{x}_{2}, \ldots, \mathbf{x}_{N}\right)$, and the resident has phenotype $\mathbf{x}$; and $r_{l}\left(\mathbf{x}_{\mathrm{m}}, \mathbf{x}\right)$ is defined as the probability that $l-1$ randomly drawn (without replacement) neighbours of a mutant are also mutants (that is, that they all belong to the same lineage). In a monomorphic population (so that $\mathbf{x}_{\mathrm{m}}=\mathbf{x}$ ), $r_{l}(\mathbf{x}, \mathbf{x})$ reduces to the probability of sampling $l$ individuals without replacement whose lineages are identical by descent, which is the standard $l^{\text {th }}$-order measure of relatedness for the island model ${ }^{51}$. The selection gradient (equation (6)) is therefore the sum of the direct fitness effects of trait $u$ and the pairwise relatedness $\left(r_{2}(\mathbf{x}, \mathbf{x})\right)$-weighted indirect fitness effects of trait $u$ (note, $\mathbf{x}_{-1}=\mathbf{x}$ means $\left.\mathbf{x}_{2}=\mathbf{x}_{3}=\cdots=\mathbf{x}_{N}=\mathbf{x}\right)^{16,19}$.

In two-dimensional phenotypic space, $\mathbf{s}(\mathbf{x})$ points towards the direction of directional selection close to the resident, so adaptive dynamics will first settle for an equilibrium

$$
\mathbf{x}^{*}=\left(z^{*}, d^{*}\right) \text { such that } \mathbf{s}\left(\mathbf{x}^{*}\right)=0
$$

when the equilibrium is an attractor of selection. The condition for the equilibrium $\mathbf{x}^{*}$ to be a local attractor depends on whether the two traits are genetically correlated. When traits are not genetically correlated (so that mutations have independent effects on both traits) and mutations affect only one trait at a time (no pleiotropy), the equilibrium is a local attractor of the evolutionary dynamic if the Jacobian matrix

$$
\mathbf{J}\left(\mathbf{x}^{\star}\right)=\left(\begin{array}{l}
\left.\left.\frac{\partial s_{z}(\mathbf{x})}{\partial z}\right|_{\mathbf{x}=\mathbf{x}^{*}} \frac{\partial s_{z}(\mathbf{x})}{\partial d}\right|_{\mathbf{x}=\mathbf{x}^{*}} \\
\left.\left.\frac{\partial s_{d}(\mathbf{x})}{\partial z}\right|_{\mathbf{x}=\mathbf{x}^{*}} \frac{\partial s_{d}(\mathbf{x})}{\partial d}\right|_{\mathbf{x}=\mathbf{x}^{*}}
\end{array}\right)
$$

has all eigenvalues with negative real parts. More generally, in the presence of pleiotropy and/or genetic correlations among traits (so that mutations have correlated effects on both traits), the equilibrium $\mathbf{x}^{\star}$ is a local attractor if the symmetric part of the Jacobian matrix $\mathbf{J}\left(\mathbf{x}^{*}\right)$ (equation (8)), 


$$
\frac{\mathbf{J}\left(\mathbf{x}^{*}\right)+\mathbf{J}\left(\mathbf{x}^{*}\right)^{\mathrm{T}}}{2}
$$

is negative-definite (that is, if it has only negative eigenvalues), and such an equilibrium is referred to as (strongly) convergence stable ${ }^{52,53}$. Note that if the symmetric part of the Jacobian matrix $\mathbf{J}\left(\mathbf{x}^{\star}\right)$ (equation (9)) is negative-definite, then $\mathbf{J}\left(\mathbf{x}^{\star}\right)$ has eigenvalues with negative real parts. For polymorphism to emerge when mutations have weak effects, it is necessary that the population is first at a convergence stable equilibrium ${ }^{54}$.

Stabilizing/disruptive selection in the infinite-island model. Once the population is at an equilibrium $\mathbf{x}^{\star}$ that is convergence stable, the greatest eigenvalue $\lambda\left(\mathbf{x}^{*}\right)$ of the Hessian matrix,

$$
\mathbf{H}\left(\mathbf{x}^{*}\right)=\left(\begin{array}{ll}
h_{z z}\left(\mathbf{x}^{*}\right) & h_{z d}\left(\mathbf{x}^{*}\right) \\
h_{z d}\left(\mathbf{x}^{*}\right) & h_{d d}\left(\mathbf{x}^{*}\right)
\end{array}\right)=\left(\begin{array}{ll}
\left.\left.\frac{\partial^{2} W\left(\mathbf{x}_{\mathrm{m}}, \mathbf{x}^{*}\right)}{\partial z_{\mathrm{m}}^{2}}\right|_{\mathbf{x}_{\mathrm{m}}=\mathbf{x}^{*}} \frac{\partial^{2} W\left(\mathbf{x}_{\mathrm{m}}, \mathbf{x}^{*}\right)}{\partial z_{\mathrm{m}} \partial d_{\mathrm{m}}}\right|_{\mathbf{x}_{\mathrm{m}}=\mathbf{x}^{*}} \\
\left.\left.\frac{\partial^{2} W\left(\mathbf{x}_{\mathrm{m}}, \mathbf{x}^{*}\right)}{\partial z_{\mathrm{m}} \partial d_{\mathrm{m}}}\right|_{\mathbf{x}_{\mathrm{m}}=\mathbf{x}^{*}} \frac{\partial^{2} W\left(\mathbf{x}_{\mathrm{m}}, \mathbf{x}^{*}\right)}{\partial d_{\mathrm{m}}^{2}}\right|_{\mathbf{x}_{\mathrm{m}}=\mathbf{x}^{*}}
\end{array}\right)
$$

tells us whether selection is stabilizing (when $\lambda\left(\mathbf{x}^{*}\right) \leq 0$ and all mutations close to the resident vanish) or disruptive (when $\lambda\left(\mathbf{x}^{*}\right)>0$ and polymorphism emerges). Note that the Hessian necessarily has real eigenvalues because it is symmetric with real entries.

In the infinite-island model, it has been shown (equation (13) of ref. ${ }^{16}$ ) that the $h_{u v}\left(\mathbf{x}^{\star}\right)$ entry of the Hessian for $u \in\{z, d\}$ and $v \in\{z, d\}$, which is the quadratic coefficient of selection on traits $u$ and $v$, can be decomposed as

$$
h_{u v}\left(\mathbf{x}^{\star}\right)=h_{\mathrm{w}, u v}\left(\mathbf{x}^{*}\right)+h_{\mathrm{r}, u v}\left(\mathbf{x}^{*}\right)
$$

where

$$
\begin{aligned}
& h_{\mathrm{w}, u v}\left(\mathbf{x}^{*}\right)=\left.\frac{\partial w\left(\mathbf{x}_{1}, \mathbf{x}_{-1}, \mathbf{x}^{*}\right)}{\partial u_{1} \partial v_{1}}\right|_{\substack{\mathbf{x}_{1}=\mathbf{x}^{*} \\
\mathbf{x}_{-1}=\mathbf{x}^{*}}} \\
& +(N-1) r_{2}\left(\mathbf{x}^{*}, \mathbf{x}^{*}\right)\left(\left.\frac{\partial^{2} w\left(\mathbf{x}_{1}, \mathbf{x}_{-1}, \mathbf{x}^{*}\right)}{\partial u_{2} \partial v_{2}}\right|_{\substack{\mathbf{x}_{1}=\mathbf{x}^{*} \\
\mathbf{x}_{-1}=\mathbf{x}^{*}}}\right. \\
& \left.+\left.\frac{\partial^{2} w\left(\mathbf{x}_{1}, \mathbf{x}_{-1}, \mathbf{x}^{*}\right)}{\partial u_{1} \partial v_{2}}\right|_{\substack{\mathbf{x}_{1}=\mathbf{x}^{*} \\
\mathbf{x}_{-1}=\mathbf{x}^{*}}}+\left.\frac{\partial^{2} w\left(\mathbf{x}_{1}, \mathbf{x}_{-1}, \mathbf{x}^{*}\right)}{\partial u_{2} \partial v_{1}}\right|_{\substack{\mathbf{x}_{1}=\mathbf{x}^{*} \\
\mathbf{x}_{-1}=\mathbf{x}^{*}}}\right) \\
& +\left.(N-1)(N-2) r_{3}\left(\mathbf{x}^{*}, \mathbf{x}^{\star}\right) \frac{\partial^{2} w\left(\mathbf{x}_{1}, \mathbf{x}_{-1}, \mathbf{x}^{*}\right)}{\partial u_{2} \partial v_{3}}\right|_{\substack{\mathbf{x}_{1}=\mathbf{x}^{*} \\
\mathbf{x}_{-1}=\mathbf{x}^{*}}}
\end{aligned}
$$

captures the second-order fitness effects when the relatedness among mutants is the same as among residents (since $r_{2}\left(\mathbf{x}^{\star}, \mathbf{x}^{\star}\right)$ and $r_{3}\left(\mathbf{x}^{*}, \mathbf{x}^{*}\right)$ are evaluated when the population is monomorphic for the resident at equilibrium), and where

$$
\begin{aligned}
h_{\mathrm{r}, u v}\left(\mathbf{x}^{*}\right)= & (N-1)\left(\left.\left.\frac{\partial w\left(\mathbf{x}_{1}, \mathbf{x}_{-1}, \mathbf{x}^{*}\right)}{\partial u_{2}}\right|_{\substack{\mathbf{x}_{1}=\mathbf{x}^{*} \\
\mathbf{x}_{-1}=\mathbf{x}^{*}}} \frac{\partial r\left(\mathbf{x}_{\mathrm{m}}, \mathbf{x}^{*}\right)}{\partial v}\right|_{\mathbf{x}_{\mathrm{m}}=\mathbf{x}^{*}}\right. \\
& \left.+\left.\left.\frac{\partial w\left(\mathbf{x}_{1}, \mathbf{x}_{-1}, \mathbf{x}^{*}\right)}{\partial v_{2}}\right|_{\substack{\mathbf{x}_{1}=\mathbf{x}^{*} \\
\mathbf{x}_{-1}=\mathbf{x}^{*}}} \frac{\partial r\left(\mathbf{x}_{\mathrm{m}}, \mathbf{x}^{*}\right)}{\partial u}\right|_{\mathbf{x}_{\mathrm{m}}=\mathbf{x}^{*}}\right)
\end{aligned}
$$

depends on the effects that the traits have on pairwise relatedness (that is, on $\partial r\left(\mathbf{x}_{\mathrm{m}}\right.$, $\left.\mathbf{x}^{\star}\right) / \partial v$ and $\left.\partial r\left(\mathbf{x}_{\mathrm{m}}, \mathbf{x}^{\star}\right) / \partial u\right)$. To evaluate this effect, it is first necessary to decompose individual fitness $w\left(\mathbf{x}_{1}, \mathbf{x}_{-1}, \mathbf{x}\right)$ as

$$
w\left(\mathbf{x}_{1}, \mathbf{x}_{-1}, \mathbf{x}\right)=w^{\mathrm{P}}\left(\mathbf{x}_{1}, \mathbf{x}_{-1}, \mathbf{x}\right)+w^{\mathrm{D}}\left(\mathbf{x}_{1}, \mathbf{x}_{-1}, \mathbf{x}\right)
$$

where $w^{\mathrm{P}}\left(\mathbf{x}_{1}, \mathbf{x}_{-1}, \mathbf{x}\right)$ is the expected number of offspring of the focal individual that remain in their natal group (that is, its expected number of philopatric offspring), and $w^{\mathrm{D}}\left(\mathbf{x}_{1}, \mathbf{x}_{-1}, \mathbf{x}\right)$ is its number of offspring that disperse. Then, for the models considered here, previous works have shown that the effect of trait $v \in\{z, d\}$ on relatedness can be expressed as

$$
\begin{aligned}
& \left.\frac{\partial r\left(\mathbf{x}_{\mathrm{m}}, \mathbf{x}^{*}\right)}{\partial v}\right|_{\mathbf{x}_{\mathrm{m}}=\mathbf{x}^{*}}=\frac{k r_{2}\left(\mathbf{x}^{\star}, \mathbf{x}^{*}\right)}{1-m\left(\mathbf{x}^{*}\right)}\left(\left(1+(N-1) r_{2}\left(\mathbf{x}^{*}, \mathbf{x}^{*}\right)\right)\right. \\
& \left.\frac{\partial w^{\mathrm{P}}\left(\mathbf{x}_{1}, \mathbf{x}_{-1}, \mathbf{x}^{*}\right)}{\partial v_{1}}\right|_{\substack{\mathbf{x}_{1}=\mathbf{x}^{*} \\
\mathbf{x}_{-1}=\mathbf{x}^{*}}} \\
& +\left(2 r_{2}\left(\mathbf{x}^{\star}, \mathbf{x}^{\star}\right)+(N-2) r_{3}\left(\mathbf{x}^{\star}, \mathbf{x}^{\star}\right)\right)
\end{aligned}
$$

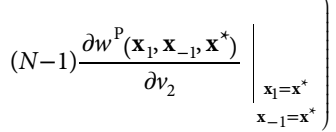

where $k$ is a constant that depends on the life cycle ( $k=2$ for the baseline WrightFisher model equation (18) of ref. ${ }^{55}$, equation (28) of ref. ${ }^{56}, k=N$ when generations overlap under the Moran model equation (14) of ref. ${ }^{16}$ ) and $m\left(\mathbf{x}^{*}\right)$ is the neutral backward probability of dispersal (that is, the probability that a breeding spot is filled by an immigrant in a population monomorphic for the resident).

The quadratic coefficient of selection on a single trait $\left(h_{z z}\left(\mathbf{x}^{\star}\right)\right.$ and $\left.h_{d d}\left(\mathbf{x}^{\star}\right)\right)$ tells us about selection on that trait when it is evolving in isolation from the other. For instance, when $h_{z z}\left(\mathbf{x}^{*}\right) \leq 0$, selection on $z$ is stabilizing, but when $h_{z z}\left(\mathbf{x}^{*}\right)>0$ selection is disruptive and $z$ will diversify whether or not dispersal is also evolving. Meanwhile, the quadratic coefficient of selection on $z$ and $d, h_{z d}\left(\mathbf{x}^{*}\right)$, captures the types of association or correlation among $z$ and $d$ that are favoured by selection. It is therefore referred to as the correlational coefficient of selection ${ }^{57}$. When $h_{z d}\left(\mathbf{x}^{*}\right)$ is positive, selection favours a positive correlation among both traits and conversely, when it is negative, selection favours a negative correlation. It follows from standard linear algebra results ${ }^{58}$, that if

$$
h_{z d}\left(\mathbf{x}^{\star}\right)^{2}>h_{z z}\left(\mathbf{x}^{\star}\right) h_{d d}\left(\mathbf{x}^{\star}\right)
$$

then the greatest eigenvalue of $\mathbf{H}\left(\mathbf{x}^{*}\right)$ is positive $\left(\lambda\left(\mathbf{x}^{*}\right)>0\right)$, which in biological terms means that if the correlational coefficient of selection is strong relative to the quadratic coefficient of selection on both traits, it causes selection to be disruptive and, hence, polymorphism. More broadly, equation (16) highlights that independently from the quadratic selection coefficients on $z$ and $d\left(h_{z z}\left(\mathbf{x}^{*}\right)\right.$ and $\left.h_{d d}\left(\mathbf{x}^{\star}\right)\right)$, the co-evolution of $z$ and $d$ always tends to promote polymorphism.

Fitness and genetic structure for the baseline model. Here, we give the necessary components to perform an invasion analysis for dispersal and benevolence under the baseline model in which generations do not overlap (that is, Wright-Fisher life cycle). First, note that the fecundity of the focal individual 1 is

$$
f\left(\mathbf{x}_{1}, \mathbf{x}_{-1}\right)=f_{0}+\sum_{j=2}^{N} \frac{\pi\left(z_{1}, z_{j}\right)}{N-1}
$$

where the payoff function $\pi$ is given in equation (2). Then, under the baseline model, the expected number of philopatric offspring of individual 1 is

$$
w^{\mathrm{P}}\left(\mathbf{x}_{1}, \mathbf{x}_{-1}, \mathbf{x}\right)=\frac{\left(1-d_{1}\right) f\left(\mathbf{x}_{1}, \mathbf{x}_{-1}\right)}{\sum_{i=1}^{N}\left(1-d_{i}\right) f\left(\mathbf{x}_{i}, \mathbf{x}_{-i}\right) / N+d\left(1-c_{\mathrm{d}}\right) f(\mathbf{x}, \mathbf{x})}
$$

and the overall fitness of individual 1 is

$$
w\left(\mathbf{x}_{1}, \mathbf{x}_{-1}, \mathbf{x}\right)=w^{\mathrm{P}}\left(\mathbf{x}_{1}, \mathbf{x}_{-1}, \mathbf{x}\right)+\frac{d_{1}\left(1-c_{\mathrm{d}}\right) f\left(\mathbf{x}_{1}, \mathbf{x}_{-1}\right)}{\left(1-d c_{\mathrm{d}}\right) f(\mathbf{x}, \mathbf{x})}
$$

To express pairwise and three-way relatedness (equations (6)-(15)), we first give the neutral backward probability of dispersal (equation (15)):

$$
m(\mathbf{x})=\frac{d\left(1-c_{\mathrm{d}}\right)}{1-d+d\left(1-c_{\mathrm{d}}\right)}
$$

which is the ratio of the number of immigrant offspring to the total number of offspring in a group. Pairwise and three-way relatedness are found using $m(\boldsymbol{x})$ and standard identity-by-descent arguments ${ }^{19,59}$ (equations (13) and (23) of ref. ${ }^{56}$ ), yielding

$$
r_{2}(\mathbf{x}, \mathbf{x})=\frac{(1-m(\mathbf{x}))^{2}}{N-(N-1)(1-m(\mathbf{x}))^{2}}
$$


and

$$
r_{3}(\mathbf{x}, \mathbf{x})=\frac{(1-m(\mathbf{x}))^{3}\left(1+3(N-1) r_{2}(\mathbf{x}, \mathbf{x})\right)}{N^{2}-(N-1)(N-2)(1-m(\mathbf{x}))^{3}}
$$

This is all that is necessary to infer the adaptive dynamics of dispersal and benevolence using the selection gradient and Hessian matrix (equations (6)-(15)). Analysis can be found in Supplementary Note 1.

Fitness and genetic structure for the baseline model when generations overlap. To incorporate generational overlap, we assume that after reproduction, a random individual in each group dies and that offspring then compete for the single open breeding spot left vacant in each group at each generation (as in a birth-death loca Moran process). In this case, fecundity and neutral backward dispersal are as above (equations (17) and (20)). However, philopatric fitness and total individual fitness are now respectively given by

$$
w^{\mathrm{P}}\left(\mathbf{x}_{1}, \mathbf{x}_{-1}, \mathbf{x}\right)=\frac{N-1}{N}+\frac{1}{N} \frac{\left(1-d_{1}\right) f\left(\mathbf{x}_{1}, \mathbf{x}_{-1}\right)}{\sum_{i=1}^{N}\left(1-d_{i}\right) f\left(\mathbf{x}_{i}, \mathbf{x}_{-i}\right) / N+d\left(1-c_{\mathrm{d}}\right) f(\mathbf{x}, \mathbf{x})}
$$

and

$$
w\left(\mathbf{x}_{1}, \mathbf{x}_{-1}, \mathbf{x}\right)=w^{\mathrm{P}}\left(\mathbf{x}_{1}, \mathbf{x}_{-1}, \mathbf{x}\right)+\frac{1}{N} \frac{d_{1}\left(1-c_{\mathrm{d}}\right) f\left(\mathbf{x}_{1}, \mathbf{x}_{-1}\right)}{\left(1-d c_{\mathrm{d}}\right) f(\mathbf{x}, \mathbf{x})}
$$

(see, for example, ref. ${ }^{16}$ ). Meanwhile, pairwise and three-way relatedness (Table 1 of ref. ${ }^{16}$ ) are given by

$$
r_{2}(\mathbf{x}, \mathbf{x})=\frac{1-m(\mathbf{x})}{1-(N-1) m(\mathbf{x})}
$$

and

$$
r_{3}(\mathbf{x}, \mathbf{x})=\frac{\left.2(1-m(\mathbf{x})) r_{2}(\mathbf{x}, \mathbf{x})\right)}{2+m(\mathbf{x})(N-2)}
$$

which completes the necessary expressions to derive the adaptive dynamics of dispersal and benevolence when generations overlap. Analysis can be found in Supplementary Note 2.

Life Sciences Reporting Summary. Further information on experimental design is available in the Life Sciences Reporting Summary.

Code availability. The Mathematica ${ }^{60} \mathrm{M}$-Files used to generate the data used in this study are available from the OSF data repository (DOI: 10.17605/OSF.IO/GBC5R).

Data availability. The data sets simulated for this study (and that appear in Figs. 3-5, and Supplementary Figs. 1 and 2) are available from the OSF data repository (DOI: 10.17605/OSF.IO/GBC5R).

Received: 4 May 2017; Accepted: 27 October 2017;

Published online: 4 December 2017

\section{References}

1. Clobert, J., Baguette, M. \& Benton, T. G. Dispersal Ecology and Evolution (Oxford Univ. Press, Oxford, 2012).

2. Ronce, O. How does it feel to be like a rolling stone? Ten questions about dispersal evolution. Annu. Rev. Ecol. Evol. Syst. 38, 231-253 (2007).

3. Hamilton, W. D. The genetical evolution of social behaviour. I. J. Theor. Biol. 7, 1-16 (1964).

4. Frank, S. A. Foundations of Social Evolution (Princeton Univ. Press, Princeton, 1998).

5. Hamilton, W. D. \& May, R. M. Dispersal in stable habitats. Nature 269, 578-581 (1977).

6. Cote, J., Clobert, J. \& Fitze, P. S. Mother-offspring competition promotes colonization success. Proc. Natl Acad. Sci. USA 104, 9703-9708 (2007).

7. Hoogland, J. L. Prairie dogs disperse when all close kin have disappeared. Science 339, 1205-1207 (2013).

8. Lehmann, L. \& Perrin, N. Altruism, dispersal, and phenotype-matching kin recognition. Am. Nat. 159, 451-468 (2002).

9. Le Galliard, J. F., Ferrière, R. \& Dieckmann, U. Adaptive evolution of social traits: origin, trajectories, and correlations of altruism and mobility. Am. Nat. 165, 206-224 (2005).

10. Hochberg, M. E., Rankin, D. J. \& Taborsky, M. The coevolution of cooperation and dispersal in social groups and its implications for the emergence of multicellularity. BMC Evol. Biol. 8, 238 (2008).
11. El Mouden, C. \& Gardner, A. Nice natives and mean migrants: the evolution of dispersal-dependent social behaviour in viscous populations. J. Evol. Biol. 21, 1480-1491 (2008).

12. Powers, S. T., Penn, A. S. \& Watson, R. A. The concurrent evolution of cooperation and the population structures that support it. Evolution 65, 1527-1543 (2011).

13. Purcell, J., Brelsford, A. \& Avilés, L. Co-evolution between sociality and dispersal: the role of synergistic cooperative benefits. J. Theor. Biol. 312, 44-54 (2012).

14. Koella, J. C. The spatial spread of altruism versus the evolutionary response of egoists. Proc. R. Soc. Lond. B 267, 1979-1985 (2000).

15. Parvinen, K. Joint evolution of altruistic cooperation and dispersal in a metapopulation of small local populations. Theor. Popul. Biol. 85, 12-19 (2013).

16. Mullon, C., Keller, L. \& Lehmann, L. Evolutionary stability of jointly evolving traits in subdivided populations. Am. Nat. 188, 175-195 (2016).

17. Wolf, M., van Doorn, G. S., Leimar, O. \& Weissing, F. J. Life-history trade-offs favour the evolution of animal personalities. Nature 447, 581-584 (2007).

18. Wright, S. Evolution in Mendelian populations. Genetics 16, 97-159 (1931).

19. Rousset, F. Genetic Structure and Selection in Subdivided Populations (Princeton Univ. Press, Princeton, 2004).

20. Dugatkin, L. A. Principles of Animal Behavior 2nd edn (WW Norton, New York, 2008).

21. Dercole, F. \& Rinaldi, S. Analysis of Evolutionary Processes: The Adaptive Dynamics Approach and its Applications (Princeton Univ. Press, Princeton, 2008).

22. Gandon, S. \& Rousset, F. Evolution of stepping-stone dispersal rates. Proc. R. Soc. Lond. B 266, 2507-2513 (1999).

23. Van Cleve, J. \& Lehmann, L. Stochastic stability and the evolution of coordination in spatially structured populations. Theor. Popul. Biol. 89, 75-87 (2013).

24. Sinervo, B. \& Svensson, E. Correlational selection and the evolution of genomic architecture. Heredity. 89, 329-338 (2002).

25. Schwander, T., Libbrecht, R. \& Keller, L. Supergenes and complex phenotypes. Curr. Biol. 24, R288-R294 (2014).

26. Leimar, O., Dall, S. R. X., Hammerstein, P. \& McNamara, J. M. Genes as cues of relatedness and social evolution in heterogeneous environments. PLoS Comput. Biol. 12, e1005006 (2016).

27. Cote, J., Clobert, J., Brodin, T., Fogarty, S. \& Sih, A. Personality-dependent dispersal: characterization, ontogeny and consequences for spatially structured populations. Phil. Trans. R. Soc. B 365, 4065-4076 (2010).

28. Wey, T. W., Spiegel, O. \& Montiglio, P. O. Natal dispersal in a social landscape: considering individual behavioral phenotypes and social environment in dispersal ecology. Curr. Zool. 61, 543-556 (2015).

29. Canestrelli, D., Bisconti, R. \& Carere, C. Bolder takes all? The behavioral dimension of biogeography. Trends Ecol. Evol. 31, 35-43 (2016).

30. Jacob, S. et al. Cooperation-mediated plasticity in dispersal and colonization. Evolution 70, 2336-2345 (2016).

31. Myers, J. H. \& Krebs, C. J. Genetic, behavioral, and reproductive attributes of dispersing field voles Microtus pennsylvanicus and Microtus ochrogaster. Ecol. Monogr. 41, 53-78 (1971).

32. Rueffler, C., Van Dooren, T. J. M., Leimar, O. \& Abrams, P. A. Disruptive selection and then what? Trends Ecol. Evol. 21, 238-245 (2006).

33. Duckworth, R. A. \& Kruuk, L. E. B. Evolution of genetic integration between dispersal and colonization ability in a bird. Evolution 63, 968-977 (2009).

34. Krackow, S. Motivational and heritable determinants of dispersal latency in wild male house mice (Mus musculus musculus). Ethology 109 671-689 (2003).

35. Rusu, A. S. \& Krackow, S. Agonistic onset marks emotional changes and dispersal propensity in wild house mouse males (Mus domesticus). J. Comp. Psychol. 119, 58-66 (2005).

36. Tung, S., Mishra, A., Gogna, N., Sadiq, M. A. \& Shreenidhi, P. M. Evolution of dispersal syndrome and its corresponding metabolomic changes. Preprint at https://www.biorxiv.org/content/early/2017/08/20/178715 (2017).

37. Bize, P., Daniel, G., Viblanc, V. A., Martin, J. G. A. \& Doligez, B. Negative phenotypic and genetic correlation between natal dispersal propensity and nest-defence behaviour in a wild bird. Biol. Lett. 13, 20170236 (2017).

38. Corcobado, G., Rodrguez-Gironés, M. A., Moya-Laraño, J. \& Avilés, L. Sociality level correlates with dispersal ability in spiders. Funct. Ecol. 26, 794-803 (2012).

39. Riechert, S. E. \& Jones, T. C. Phenotypic variation in the social behaviour of the spider Anelosimus studiosus along a latitudinal gradient. Anim. Behav. 75, 1893-1902 (2008).

40. Pruitt, J. N. \& Riechert, S. E. Sex matters: sexually dimorphic fitness consequences of a behavioural syndrome. Anim. Behav. 78, 175-181 (2009).

41. Pruitt, J. N. et al. Population differences in behaviour are explained by shared within-population trait correlations. J. Evol. Biol. 23, 748-756 (2010).

42. Keller, L. et al. Ecology and evolution of social organization: insight from fire ants and other highly eusocial insects. Annu. Rev. Ecol. Syst. 26, 631-656 (1995) 
43. Purcell, J. \& Chapuisat, M. Bidirectional shifts in colony queen number in a socially polymorphic ant population. Evolution 67, 1169-1180 (2013).

44. Wang, J. et al. A Y-like social chromosome causes alternative colony organization in fire ants. Nature 493, 664-668 (2013).

45. Purcell, J., Brelsford, A., Wurm, Y., Perrin, N. \& Chapuisat, M. Convergent genetic architecture underlies social organization in ants. Curr. Biol. 24, 2728-2732 (2014)

46. Clutton-Brock, T. H. et al. Infanticide and expulsion of females in a cooperative mammal. Proc. R. Soc. Lond. B 265, 2291-2295 (1998).

47. Bohonak, A. J. Dispersal, gene flow, and population structure. Theor. Popul. Biol. 74, 21-45 (1999).

48. Lehmann, L. \& Keller, L. The evolution of cooperation and altruism-a general framework and a classification of models. J. Evol. Biol. 19, 1365-1376 (2006).

49. Bulmer, M. G. Sex ratio theory in geographically structured populations. Heredity 56, 69-73 (1986).

50. Lessard, S. Evolutionary stability: one concept, several meanings. Theor. Popul. Biol. 37, 159-170 (1990).

51. Roze, D. \& Rousset, F. Multilocus models in the infinite island model of population structure. Theor. Popul. Biol. 73, 529-542 (2008).

52. Leimar, $\mathrm{O}$. The evolution of phenotypic polymorphism: randomized strategies versus evolutionary branching. Am. Nat. 165, 669-681 (2005).

53. Leimar, O. Multidimensional convergence stability. Evol. Ecol. Res. 11, 191-208 (2009).

54. Geritz, S. A. H., Metz, J. A. J. \& Rueffler, C. Mutual invadability near evolutionarily singular strategies for multivariate traits, with special reference to the strongly convergence stable case. J. Math. Biol. 72, 1081-1099 (2016).

55. Ajar, E. Analysis of disruptive selection in subdivided populations. BMC Evol. Biol. 3, 22 (2003).
56. Wakano, J. Y. \& Lehmann, L. Evolutionary branching in deme-structured populations. J. Theor. Biol. 351, 83-95 (2014).

57. Phillips, P. C. \& Arnold, S. J. Visualizing multivariate selection. Evolution 43 1209-1222 (1989).

58. Bhatia, R. Positive Definite Matrices Vol. 53 (Princeton Univ. Press, Princeton, 2013).

59. Karlin, S. Equilibrium behaviour of population genetic models with non-random mating. J. Appl. Probab. 5, 231-313 (1968).

60. Mathematica (Wolfram Research, Champaign, 2014).

\section{Author contributions}

C.M., L.K. and L.L. conceptualized the study. C.M. designed the models and performed the analyses. C.M., L.K. and L.L. wrote the manuscript.

\section{Competing interests}

The authors declare no competing financial interests.

\section{Additional information}

Supplementary information is available for this paper at https://doi.org/10.1038/ s41559-017-0397-y.

Reprints and permissions information is available at www.nature.com/reprints. Correspondence and requests for materials should be addressed to C.M. or L.K. Publisher's note: Springer Nature remains neutral with regard to jurisdictional claims in published maps and institutional affiliations. 


\section{natureresearch}

\section{Life Sciences Reporting Summary}

Nature Research wishes to improve the reproducibility of the work that we publish. This form is intended for publication with all accepted life science papers and provides structure for consistency and transparency in reporting. Every life science submission will use this form; some list items might not apply to an individual manuscript, but all fields must be completed for clarity.

For further information on the points included in this form, see Reporting Life Sciences Research. For further information on Nature Research policies, including our data availability policy, see Authors \& Referees and the Editorial Policy Checklist.

\section{- Experimental design}

\section{Sample size}

Describe how sample size was determined.

2. Data exclusions

Describe any data exclusions.

3. Replication

Describe whether the experimental findings were reliably reproduced.

4. Randomization

Describe how samples/organisms/participants were allocated into experimental groups.

5. Blinding

Describe whether the investigators were blinded to group allocation during data collection and/or analysis.
We simulated evolution in populations of at least $10^{\prime} 000$ individuals as previous studies (e.g. Wakano Genetics 2012, Wakano Lehmann TPB 2014) have shown that such populations behave almost deterministically (i.e., genetic drift plays a minimal role in evolution).

Note: all studies involving animals and/or human research participants must disclose whether blinding and randomization were used.

\section{Statistical parameters}

For all figures and tables that use statistical methods, confirm that the following items are present in relevant figure legends (or in the Methods section if additional space is needed).

$\mathrm{n} / \mathrm{a}$ Confirmed

\The exact sample size $(n)$ for each experimental group/condition, given as a discrete number and unit of measurement (animals, litters, cultures, etc.)

$\triangle$ A description of how samples were collected, noting whether measurements were taken from distinct samples or whether the same

sample was measured repeatedly

$\bigotimes$ A statement indicating how many times each experiment was replicated

The statistical test(s) used and whether they are one- or two-sided (note: only common tests should be described solely by name; more complex techniques should be described in the Methods section)

Х $\square$ A description of any assumptions or corrections, such as an adjustment for multiple comparisons

Х $\square$ The test results (e.g. $P$ values) given as exact values whenever possible and with confidence intervals noted

$\bigotimes$ A clear description of statistics including central tendency (e.g. median, mean) and variation (e.g. standard deviation, interquartile range)

$\square$ \Clearly defined error bars 
Policy information about availability of computer code

\section{Software}

Describe the software used to analyze the data in this study.

We used Wolfram Mathematica version 10.2.0.0.

For manuscripts utilizing custom algorithms or software that are central to the paper but not yet described in the published literature, software must be made available to editors and reviewers upon request. We strongly encourage code deposition in a community repository (e.g. GitHub). Nature Methods guidance for providing algorithms and software for publication provides further information on this topic.

\section{- Materials and reagents}

Policy information about availability of materials

\section{Materials availability}

Indicate whether there are restrictions on availability of unique materials or if these materials are only available for distribution by a for-profit company.

\section{Antibodies}

Describe the antibodies used and how they were validated for use in the system under study (i.e. assay and species).

10. Eukaryotic cell lines

a. State the source of each eukaryotic cell line used.

b. Describe the method of cell line authentication used.

c. Report whether the cell lines were tested for mycoplasma contamination.

d. If any of the cell lines used are listed in the database of commonly misidentified cell lines maintained by ICLAC, provide a scientific rationale for their use.
No unique materials were used.

No antibodies were used.

No eukaryotic cell lines were used.

No eukaryotic cell lines were used.

No eukaryotic cell lines were used.

No commonly misidentified cell lines were used.

\section{- Animals and human research participants}

Policy information about studies involving animals; when reporting animal research, follow the ARRIVE guidelines

\section{Description of research animals}

Provide details on animals and/or animal-derived materials used in the study.
No animals were used.

Policy information about studies involving human research participants

\section{Description of human research participants}

Describe the covariate-relevant population characteristics of the human research participants.
The study did not involve human research participants. 